\title{
Efficacy and safety of selective laser trabeculoplasty and pattern scanning laser trabeculoplasty: a randomised clinical trial
}

\author{
Mandy Oi Man Wong, ${ }^{1,2}$ Isabel SW Lai, ${ }^{1,2}$ Poemen Puiman Chan (D) 1,2 \\ Noel CY Chan, ${ }^{1,3}$ Alison YY Chan, ${ }^{1,2}$ Gilda WK Lai, ${ }^{1}$ Vivian SM Chiu, ${ }^{1}$ \\ Christopher Kai-Shun Leung (D) 1,2
}

- Additional material is published online only. To view please visit the journal online (http://dx.doi.org/10.1136/ bjophthalmol-2020-316178).

'Department of Ophthalmology and Visual Sciences, The Chinese University of Hong Kong, Shatin, Hong Kong

${ }^{2}$ Department of Ophthalmology, Hong Kong Eye Hospital, Kowloon City, Hong Kong ${ }^{3}$ Department of Ophthalmology \& Visual Sciences, Prince of Wales Hospital, Shatin, Hong Kong

Correspondence to

Dr Christopher Kai-Shun Leung, The Chinese University of Hong Kong, Hong Kong, Hong Kong; cksleung@gmail.com

MOMW and ISL contributed equally.

Received 29 February 2020

Revised 6 May 2020

Accepted 23 May 2020

Published Online First

30 June 2020

Check for updates

(C) Author(s) (or their employer(s)) 2021. No commercial re-use. See rights and permissions. Published by BMJ.

To cite: Wong MOM,

Lai ISW, Chan PP, et al.

$\mathrm{Br} J$ Ophthalmol

2021:105:514-520.

\section{ABSTRACT}

Aims To compare the intraocular pressure (IOP) lowering effect and safety profile between pattern scanning laser trabeculoplasty (PSLT) and selective laser trabeculoplasty $(\mathrm{SLT})$ in patients with primary open-angle glaucoma (POAG) or ocular hypertension (OHT) over a 12-month follow-up.

Methods 132 patients with POAG or OHT were consecutively enrolled and randomised (1:1) to receive PSLT $(n=65)$ or SLT $(n=67)$ in a single centre. IOP was measured before and then on 1 day, 1 week, 1, 3, 6, 9 and 12 months after PSLT/SLT. The primary outcome measure was the proportion of patients with $\geq 20 \%$ IOP reduction at 12 months without IOP-lowering medications (complete success).

Results The mean baseline IOP was $21.2 \pm 4.1 \mathrm{~mm} \mathrm{Hg}$ for eyes randomised to PSLT and $21.3 \pm 4.7 \mathrm{~mm} \mathrm{Hg}$ for eyes randomised to SLT ( $p=0.898)$. At 12 months, the IOP was $18.3 \pm 3.1$ and $17.8 \pm 3.4 \mathrm{~mm} \mathrm{Hg}$, respectively $(p=0.402)$. IOP measurements were comparable between the groups over 12 months (overall mean difference $0.4 \mathrm{~mm} \mathrm{Hg}, 95 \% \mathrm{Cl}:-0.5$ to $1.3 \mathrm{~mm} \mathrm{Hg}$ ). $15.4 \%$ of PSLT-treated and $25.4 \%$ of SLT-treated patients achieved treatment success (difference: $10.0 \%, 95 \% \mathrm{Cl}:-3.6$ to 23.6) $(p=0.155)$, respectively. A higher baseline IOP and a greater percentage of IOP reduction at day 1 were associated with a greater percentage of IOP reduction at 12 months $(p<0.001)$. There were no significant differences in visual field mean deviation, average retinal nerve fibre layer thickness, corneal endothelial cell count and visual acuity between the treatment groups at the baseline and 12-month follow-up ( $p \geq 0.062$ ).

Conclusions PSLT was not superior to SLT in terms of safety and IOP-lowering efficacy in patients with POAG or $\mathrm{OHT}$.

Trial registration number The clinical trial was registered in the Centre for Research and Biostatistics Clinical Trials Registry, the Chinese University of Hong Kong (Identifier CUHK_CCT00407). The full trial protocol can be accessed from the authors on request.

Whereas selective laser trabeculoplasty (SLT) has been recommended as a first-line treatment option to lower intraocular pressure (IOP) in patients with open angle glaucoma, ${ }^{1}$ the safety and efficacy of pattern scanning laser trabeculoplasty (PSLT) are less studied. Pattern scanning laser trabeculoplasty shares a similar principle with SLT-selective photothermolysis leading to trabecular meshwork remodelling and an increase in outflow facility. ${ }^{2-4}$ The main difference between PSLT and SLT rests on the amount of treatment energy delivered onto the trabecular meshwork (table 1). SLT delivers approximately 4.8 to 9.5 $\mathrm{mJ}$ per $\mathrm{mm}^{2}$ (with a treatment spot size of $400 \mu \mathrm{m}$ and assuming each application delivers 0.6 to $1.2 \mathrm{~mJ}$ ) whereas PSLT delivers approximately 318 to $446 \mathrm{~mJ}$ per $\mathrm{mm}^{2}$ (with a treatment spot size of $100 \mu \mathrm{m}$ and assuming each application delivers 2.5 to $3.5 \mathrm{~mJ}$ ). The higher energy delivered by PSLT does not appear to induce more intraocular inflammation compared with SLT. In an early study investigating the clinical application of PSLT in 25 glaucoma patients, Turati and colleagues reported no IOP spikes and no inflammation following PSLT and the IOP reduction was $24 \%$ at 6 months. ${ }^{5}$ The treatment duration for PSLT is shorter than SLT; instead of applying 100 individual laser spots for $360^{\circ}$ treatment in SLT, PSLT automatically rotates the aiming beam and delivers multiple laser spots over 32 segments (each segment spans $\sim 11.25^{\circ}$ ) of the trabecular meshwork for $360^{\circ}$ treatment. With a higher energy delivered by PSLT compared with SLT, PSLT might offer a greater IOPlowering effect. In a recent randomised controlled trial comparing PSLT and SLT in 29 patients with open-angle glaucoma over 6 months of follow-up, Mansouri and Shaarawy showed that eyes treated with PSLT attained a higher patient-reported comfort level and a greater percentage of IOP reduction at 1 month and 3 months compared with SLT $(p<0.01){ }^{6}$ Although the percentage of IOP reduction remained greater for PSLT than SLT at 6 months, the difference was not statistically significant (the sample size of the study was calculated to detect IOP difference between the treatment groups at 1 month). A longer-term study is therefore imperative to address if PSLT provides an additional IOP-lowering benefit compared with SLT. In this 1 year prospective randomised clinical trial, we compared the treatment outcomes in 132 patients with primary open-angle glaucoma (POAG) or ocular hypertension (OHT). Further, we investigated factors associated with treatment success of PSLT and SLT.

\section{METHODS}

\section{Study design}

This is a 1 year, prospective, randomised, singlesite, parallel-group clinical trial comparing the safety and IOP-lowering efficacy of PSLT (PASCAL Streamline 577, Topcon) and SLT (Selecta II SLT, Lumenis) in patients with POAG or OHT. The flow 
Table 1 Comparison of specifications and energy settings between pattern scanning laser trabeculoplasty and selective laser trabeculoplasty

\begin{tabular}{lll}
\hline & $\begin{array}{l}\text { Pattern scanning laser } \\
\text { trabeculoplasty }\end{array}$ & $\begin{array}{l}\text { Selective } \\
\text { lasertrabeculoplasty }\end{array}$ \\
\hline Laser wavelength & $\begin{array}{l}\text { Optically pumped } \\
\text { semiconductor laser } 577 \mathrm{~nm}\end{array}$ & $\begin{array}{l}\text { Q-switched, frequency- } \\
\text { doubled, Nd:YAG laser } \\
532 \mathrm{~nm}\end{array}$ \\
\hline $\begin{array}{l}\text { Laser spot diameter } \\
\text { Laser energy per pulse }\end{array}$ & $\mathbf{1 0 0 \mu \mathrm { m }}$ & $400 \mu \mathrm{m}$ \\
\hline $\begin{array}{l}\text { Laser pulse length } \\
\text { Energy delivered per } \mathrm{mm}^{2}\end{array}$ & $5 \mathrm{~ms} \mathrm{~mJ}$ & $0.6-1.2 \mathrm{~mJ}$ \\
\hline $\begin{array}{l}\text { *Number of applications } \\
\text { for } 360^{\circ} \text { treatment }\end{array}$ & 1248 & $3 \mathrm{~ns}$ \\
\hline $\begin{array}{l}\text { Total energy delivered for } \\
360^{\circ} \text { treatment }\end{array}$ & $3120-4368 \mathrm{~mJ}$ & $4.8-9.5 \mathrm{~mJ}$ \\
\hline
\end{tabular}

${ }^{*}$ The number of laser applications is fixed at 1248 for $360^{\circ}$ of trabecular meshwork treatment with pattern scanning laser trabeculoplasty whereas the number of laser applications may vary for $360^{\circ}$ of trabecular meshwork treatment with selective laser trabeculoplasty.

chart of study design is shown in figure 1 . Written informed consent was obtained from all study participants. Patients were recruited from the general clinic and the glaucoma clinic in Hong Kong Eye Hospital between January 2015 and December
2016. Last patient follow-up was completed in January 2018. The study was conducted according to the CONSORT (Consolidated Standards of Reporting Trials) 2010 guidelines (please refer to the Appendix for the checklist).

\section{Subjects}

Consecutive patients who were older than 18 years of age, had a diagnosis of POAG or OHT with trabecular meshwork visible for 360 degrees and an IOP $\leq 35 \mathrm{~mm} \mathrm{Hg}$ (after washout) were eligible for inclusion. Patients with POAG had a narrowed neuroretinal rim and/or a thinned retinal nerve fibre layer (RNFL) in clinical examination or in the optical coherence tomography (OCT) RNFL thickness analysis (described below) with no identifiable secondary causes. Patients with glaucomatous optic disc changes but without visual field defects (ie, pre-perimetric glaucoma) were eligible for inclusion. Ocular hypertension was defined based on the detection of IOP $>21 \mathrm{~mm} \mathrm{Hg}$ in three follow-up visits without evidence of glaucoma.

\section{Screening visit}

At the screening visit, visual acuity and refraction were measured, dark room gonioscopy, slit-lamp biomicroscopy, dilated fundus and optic disc examination were performed. RNFL thickness was measured with OCT (Cirrus HD-OCT, Carl Zeiss Meditec,

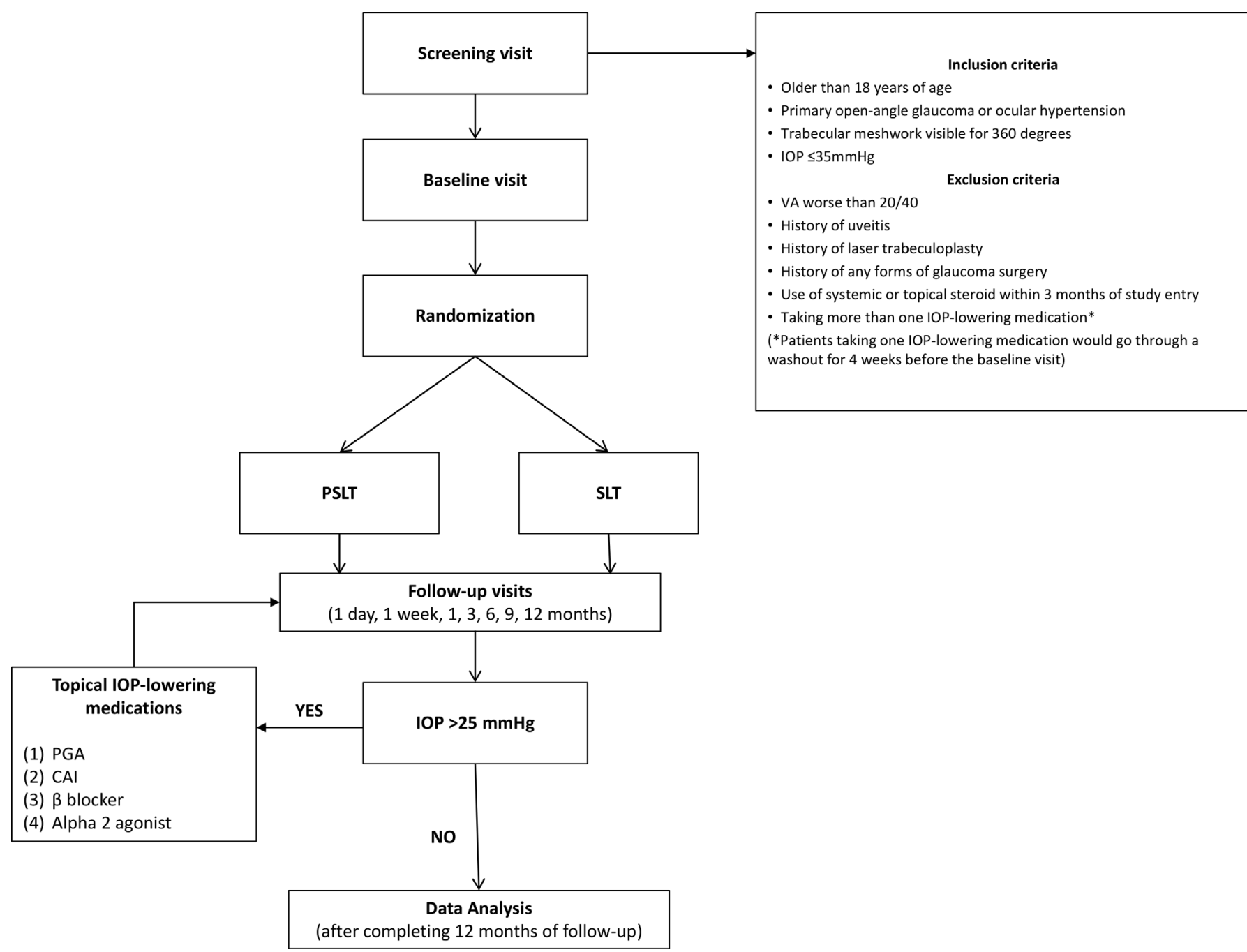

Figure 1 Study design flow chart. IOP, intraocular pressure; CAI, carbonic anhydrase inhibitor; PGA, prostaglandin analogue; PSLT,pattern scanning laser trabeculoplasty; SLT, selective laser trabeculoplasty; VA, visual acuity. 
Dublin, California) and visual field sensitivity was measured with standard automated perimetry (Humphrey Field Analyzer 740i, Carl Zeiss Meditec). Patients were excluded if the visual acuity was worse than 20/40, if there was history of uveitis, laser trabeculoplasty, any form of glaucoma surgery, systemic or topical use of steroid within 3 months of study entry and if they were using more than one class of IOP-lowering medication. Patients who were taking one class of IOP-lowering medication with an IOP $<22 \mathrm{~mm} \mathrm{Hg}$ would go through a washout period of 4 weeks before baseline IOP measurements. Any patients with POAG or OHT meeting the study criteria were eligible for laser treatment. They were randomised to receive PSLT or SLT within 4 weeks of baseline IOP measurements. At the time of PSLT or SLT, patients were not taking any IOP-lowering medication. They were then followed up at 1 day, 1 week, 1, 3, 6, 9 and 12 months.

\section{Randomisation}

Randomisation (1:1) to PSLT or SLT was carried out based on an odd/even designation generated from a random number table masked to the investigators except the technician (GL) responsible for the randomisation. If both eyes of a patient were eligible for inclusion, only one eye would be assigned for randomisation according to an alternate right and left sequence except for patients with visual field defects detected in only one eye-the eye with visual field defects was randomised. The patients, but not the ophthalmologists, were masked to treatment allocation. PSLT/SLT was performed by five trained ophthalmologists (MW, IL, PC, NC and CL).

\section{Baseline and follow-up IOP measurements and investigations} Intraocular pressure was measured at the baseline, and then at 1 day, 1 week, 1, 3, 6, 9 and 12 months after PSLT or SLT, using a calibrated Goldmann applanation tonometer by trained ophthalmologists masked to the clinical information. Two readings were obtained for each eye and the average was computed for analysis. If the difference of the two measurements were greater than $2 \mathrm{~mm} \mathrm{Hg}$, a third reading would be recorded, and the median was used in the analysis. The baseline, 6 month and 12 month IOP measurements were obtained from taking the average of three IOP readings captured at $9 \mathrm{am}, 1 \mathrm{pm}$ and $5 \mathrm{pm}$ on the same day. Visual field sensitivity, OCT RNFL thickness, corneal endothelial cell count and visual acuity were measured at the baseline, 6 month and 12 month visits.

\section{Visual field testing, RNFL imaging and specular microscopy}

Visual field testing was performed using the Humphrey Field Analyzer II-i (Carl Zeiss Meditec). All visual fields had fixation losses less than 20\%, and false positive errors less than $15 \%$. Average visual field sensitivity was expressed in mean deviation (MD). The RNFL thickness was measured by the Cirrus HD-OCT (Carl Zeiss Meditec) using the optic disc cube scan which imaged the RNFL in a $6 \times 6 \mathrm{~mm}^{2}$ region $(200 \times 200$ pixels $)$ centred at the optic disc to generate the RNFL thickness map and the circumpapillary average RNFL thickness. Glaucomatous RNFL defects were defined by a score of 5 in the RNFL deviation map as described in our previous study. ${ }^{7}$ Only images with a signal strength $\geq 7$ were included. Images with motion artefact, poor concentration, poor focus or missing data were detected by the operator at the time of imaging with re-scanning performed in the same visit. Corneal endothelial cell count was measured with specular microscopy (Specular Microscope SP-8000, Konan Medical Inc, Japan).

\section{Pattern scanning laser trabeculoplasty}

Pattern scanning laser trabeculoplasty was performed with the PASCAL Streamline 577 (Topcon, Tokyo, Japan). A drop of $0.1 \%$ brimonidine was instilled into the eye 15 min prior to treatment. After instilling a drop of $0.5 \%$ proparacaine, the Latina $1 \mathrm{X}$ lens (Ocular Instruments, Bellevue, Washington) with coupling fluid was then attached to the cornea and the trabecular meshwork was visualised. The laser power required for treatment was determined during the titration mode. A single pulse of laser (duration: $10 \mathrm{~ms}$; size: $100 \mu \mathrm{m}$; power: starting from $500 \mathrm{~mW}$ ) was applied with the energy titrated to a level until the inferior trabecular meshwork is lightly blanched. During the treatment, the laser power was maintained but the duration was shortened to $5 \mathrm{~ms}$. PSLT delivered a total of 1248 pulses in 32 sequential segments. Each segment comprised 39 spots (13 columns x 3 rows) spanning $11.25^{\circ}$ of the trabecular meshwork. The contact lens was rotated every $11.25^{\circ}$ after a segment was treated and the procedure was repeated 32 times so that the trabecular meshwork was treated for $360^{\circ}$ with no overlap and no gap of the treatment spots.

\section{Selective laser trabeculoplasty}

Selective laser trabeculoplasty was performed with Selecta II SLT (Lumenis, Israel). A drop of $0.1 \%$ brimonidine was instilled into the eye 15 min prior to treatment. After instilling a drop of $0.5 \%$ proparacaine, the Latina $1 \mathrm{X}$ lens with coupling fluid was then attached to the cornea and the trabecular meshwork was visualised. The laser spot size was $400 \mu \mathrm{m}$ and the treatment duration was $3 \mathrm{~ns}$. The energy was increased by $0.1 \mathrm{~mJ}$ from $0.6 \mathrm{~mJ}$ until champagne-like bubbles were formed on the inferior trabecular meshwork. The energy was then reduced by $0.1 \mathrm{~mJ}$ for the rest of treatment. The contact lens was rotated until the trabecular meshwork was treated for $360^{\circ}$ with approximately 100 nonoverlapping shots.

\section{Additional treatments}

One topical IOP-lowering medication (prostaglandin analogue, beta blocker, carbonic anhydrase inhibitor or alpha 2-adrenergic agonist) would be prescribed if the IOP was greater than $25 \mathrm{~mm}$ $\mathrm{Hg}$ during the study follow-up and there were no contraindications (in the original study design, topical IOP-lowering treatment would be provided if the IOP is higher than $30 \mathrm{~mm} \mathrm{Hg}$; re-treatment with the same type of laser trabeculoplasty would be performed at 6 months if the IOP cannot be reduced by $20 \%$ from baseline. We then adopted a revised protocol and provided topical IOP-lowering treatment if the IOP was higher than $25 \mathrm{~mm}$ $\mathrm{Hg}$; no patients received re-treatment of PSLT/SLT throughout the study period). Additional topical IOP-lowering medication, one class at a time, would be given if the IOP remained higher than $25 \mathrm{~mm} \mathrm{Hg}$ in the subsequent follow-up visits. The fellow eye was treated at the discretion of the attending ophthalmologists.

\section{Outcome measures}

The primary outcome measure was complete success, defined as $\geq 20 \%$ IOP reduction from the baseline at 12 months without IOP-lowering medications. Partial success was defined when the IOP was reduced by $\geq 20 \%$ from baseline at 12 months with or without IOP-lowering medications. Other outcome measures included the overall mean IOP measured from 1 day, 1 week, 1 month, 3 months, 6 months, 9 months and 12 months, the number of IOP-lowering medications, visual field MD, average RNFL thickness, visual acuity (the original plan included measurement of cataract density but it was not performed) and 
corneal endothelial cell counts at 12 months. Complications including significant IOP elevation ( $\geq 20 \%$ from the baseline) and anterior chamber inflammation were recorded during the follow-up visits. Factors (age, baseline IOP, percentage of IOP reduction at day 1 , baseline visual field $\mathrm{MD}$ and number of IOP-lowering medication at 12 months) associated with the percentage of IOP reduction at 12 months were identified.

\section{Statistics}

Statistical analysis was performed with Stata (StataCorp, College Station, Texas, V.15.0). Treatment success in studies of SLT is typically defined by $\geq 20 \%$ IOP reduction or $\geq 3 \mathrm{~mm} \mathrm{Hg}$ IOP reduction. ${ }^{8}$ With reference to a study reporting treatment success (IOP reduction $\geq 20 \%$ ) at 12 month to be $60 \%$ for patients with open-angle glaucoma, ${ }^{10}$ we estimated that at least 112 patients, 56 patients for each treatment arm, would be needed to detect a difference of $25 \%$ in the proportion of patients with treatment success between SLT and PSLT at an alpha of 5\% and a beta of $85 \%$, with a hypothesis of PSLT to be more effective than SLT in lowering IOP. Assuming an attrition rate of $15 \%$, we recruited 132 POAG patients. Data were analysed on an intention-to-treat basis. The proportions of patients attaining treatment success and the proportions of patients on IOP-lowering medication at 12 months were compared with $\chi^{2}$ test. The difference in overall mean IOP measurements over the 1-year follow-up (baseline, 1 day, 1 week, 1, 3, 6, 9 and 12 months) between the treatment arms was compared with a linear mixed model. The differences in IOP at individual follow-up visits; the differences in visual field MD, average RNFL thickness, corneal endothelial cell count and visual acuity between the treatment arms at the baseline and 12 month follow-up were compared with independent t-test. Factors associated with the percentage of IOP reduction at 12 months were investigated with multivariable linear regression. P value $<0.05$ was considered statistically significant.

\section{RESULTS}

One hundred and forty-nine potentially eligible patients were examined consecutively at Hong Kong Eye Hospital. After excluding 17 patients who did not meet the inclusion criteria or declined to participate in the study, 132 eyes from 132 patients with POAG (110 patients, $83.3 \%$ ) or OHT (22 patients, $16.7 \%$ ) were included. Their baseline IOP was $20.6 \pm 4.4 \mathrm{~mm} \mathrm{Hg}$ and $24.6 \pm 2.5 \mathrm{~mm} \mathrm{Hg}$, respectively. For those with POAG $(\mathrm{n}=110)$, the mean visual field $\mathrm{MD}$ at the baseline was $-6.1 \pm 4.6 \mathrm{~dB} ; 59$ eyes $(53.6 \%)$ had visual field $\mathrm{MD} \geq-6 \mathrm{~dB}$. The 132 patients were randomised to receive SLT (67 patients) or PSLT (65 patients) (figure 2). The mean IOP was $21.3 \pm 4.7 \mathrm{~mm} \mathrm{Hg}$ for eyes randomised to SLT, and $21.2 \pm 4.1 \mathrm{~mm} \mathrm{Hg}$ for eyes randomised to PSLT. At the baseline visit, 65 patients (49.2\%) had mean IOP $\leq 21 \mathrm{~mm} \mathrm{Hg}$. There were no significant differences in IOP, age, spherical equivalent, visual field MD, average RNFL thickness and visual acuity between the treatment arms at the baseline visit (online supplementary table 1).

\section{Treatment effect of SLT/PSLT: 12-month follow-up}

The IOP measurements were comparable between the treatment arms over 1 year of follow-up with an overall mean difference of $0.4 \mathrm{~mm} \mathrm{Hg}(95 \% \mathrm{CI}:-0.5$ to $1.3 \mathrm{~mm} \mathrm{Hg}, \mathrm{p}=0.384$, linear mixed effects modelling) after adjusting for age and number of glaucoma medications applied during follow-up (figure 3). IOP measurements between the SLT-treated and PLT-treated groups were comparable at the individual follow-up visits except for day 1 at which SLT-treated eyes had lower IOP $(13.6 \pm 4.1 \mathrm{~mm} \mathrm{Hg})$ than PSLT-treated eyes $(15.4 \pm 3.4 \mathrm{~mm} \mathrm{Hg})(p=0.006)$. At 12 months, the mean IOP reduction from baseline was 14.7\% (95\% CI: $11.2 \%$ to $18.1 \%)$ for eyes treated with SLT and $11.6 \%(95 \%$ CI: $7.4 \%$ to $15.9 \%)$ for eyes treated with PSLT $(p=0.271)$; 50 patients $(74.6 \%)$ treated with SLT and 47 patients $(72.3 \%)$

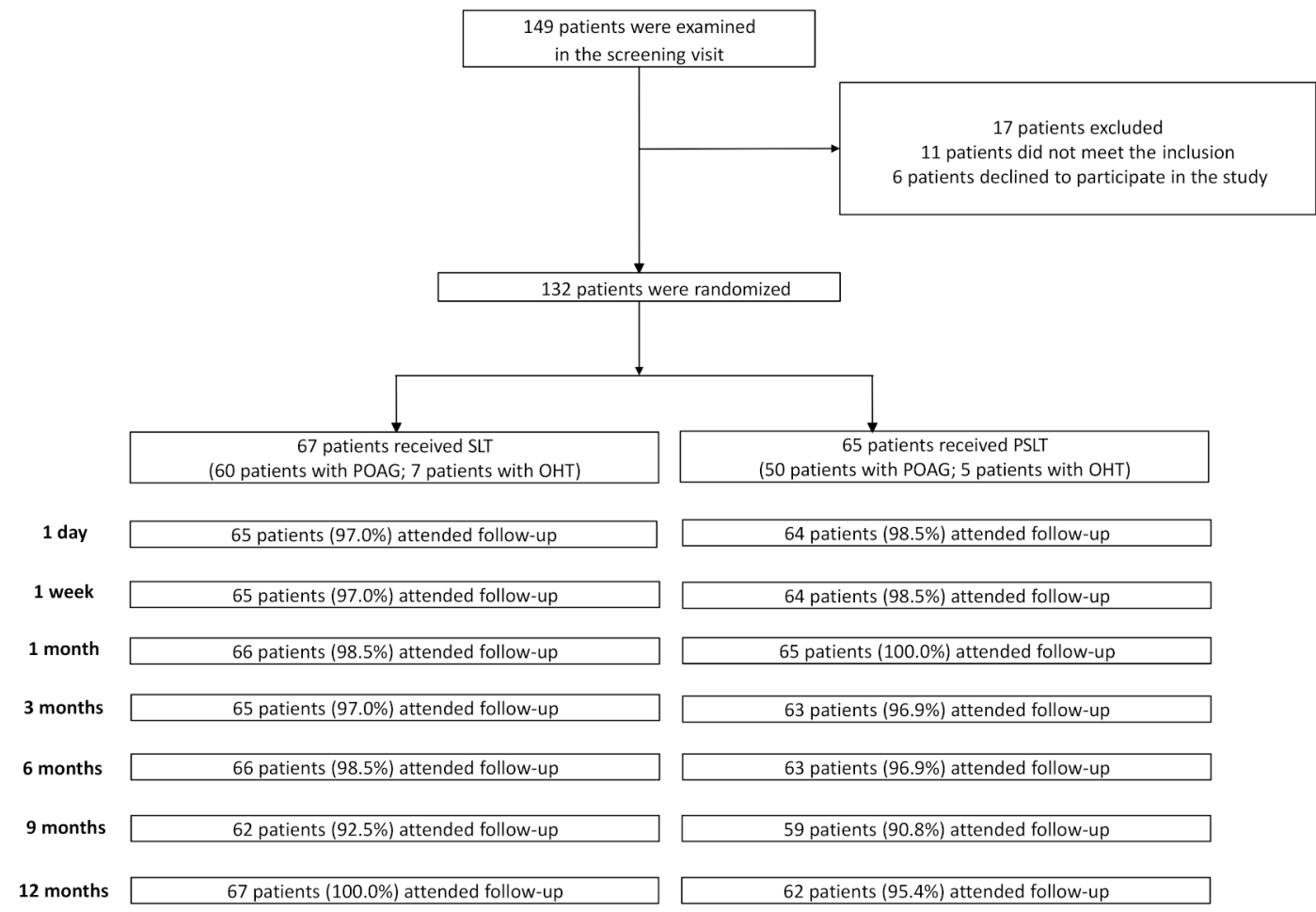

Figure 2 Trial profile. OHT, ocular hypertension; POAG, primary open-angle glaucoma; PSLT, patternscanning laser trabeculoplasty; SLT, selectivelaser trabeculoplasty. 


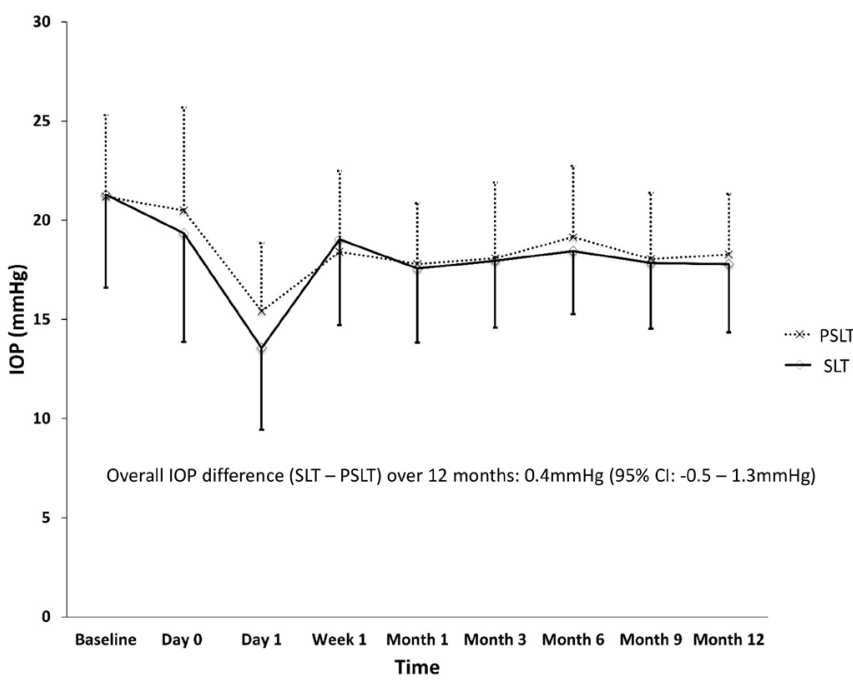

\begin{tabular}{cccc}
\hline \hline & PSLT $($ mean \pm SD $)$ & SLT $($ mean \pm SD $)$ & P \\
\hline Baseline & $21.2 \pm 4.1$ & $21.3 \pm 4.7$ & 0.898 \\
Day 1 & $15.4 \pm 3.4$ & $13.6 \pm 4.1$ & 0.006 \\
Week 1 & $18.4 \pm 4.1$ & $19.0 \pm 4.3$ & 0.392 \\
Month 1 & $17.8 \pm 3.1$ & $17.6 \pm 3.7$ & 0.706 \\
Month 3 & $18.1 \pm 3.8$ & $18.0 \pm 3.4$ & 0.837 \\
Month 6 & $19.1 \pm 3.6$ & $18.4 \pm 3.2$ & 0.230 \\
Month 9 & $18.0 \pm 3.3$ & $17.8 \pm 3.3$ & 0.736 \\
Month 12 & $18.3 \pm 3.1$ & $17.8 \pm 3.4$ & 0.402 \\
\hline
\end{tabular}

Figure 3 Comparisons of intraocular pressure (IOP) measurements between pattern scanning laser trabeculoplasty (PSLT) and selective laser trabeculoplasty (SLT). Error bars: standard deviation (SD)

treated with PSLT remained medication-free $(p=0.764)$. In patients who were medication-free at 12 months, the mean IOP reduction from baseline at 12 months was 12.0\% (95\% CI: 8.1\% to $15.9 \%$ ) for those treated with SLT and $8.4 \%$ (95\% CI: $3.6 \%$ to $13.2 \%)$ for those treated with PSLT $(\mathrm{p}=0.237)$. The proportions of eyes attaining complete treatment success were $25.4 \%$ (95\% CI: $14.9 \%$ to $35.8 \%$ ) (17 patients) in the SLT-treated group, and $15.4 \%$ (95\% CI: $6.6 \%$ to $24.1 \%$ ) (10 patients) in the PSLT-treated group (difference in proportion: 10.0\%, 95\% CI: -3.6 to $23.6 \% ; \mathrm{p}=0.155)$ and $40.3 \%$ (95\% CI: $28.6 \%$ to $52.0 \%$ ) (27 patients) and 35.4\% (95\% CI: $23.8 \%$ to $47.0 \%$ ) (23 patients) of eyes attained partial treatment success, respectively (difference in proportion: 4.9\%, 95\% CI: -11.6 to $21.4 \%$; $\mathrm{p}=0.561$ ).

\section{Factors associated with the degree of IOP reduction at 12 months}

In the multivariable linear regression models (online supplementary table 2), a higher baseline IOP (every mm Hg increase) (figure 4A), a greater percentage of IOP reduction at day 1 (every $10 \%$ decrease in IOP from baseline) (figure 4B) and a greater number of items of topical IOP-lowering medications used at 12 months (every item increase in IOP-lowering medications) were associated with 1.7\% (95\% CI: $1.2 \%$ to $2.3 \%$ ), $2.9 \%$ (95\% CI: $1.6 \%$ to $4.3 \%$ ) and $4.0 \%$ (95\% CI: $0.5 \%$ to $7.6 \%$ ) greater IOP reduction at 12 months, respectively, after adjusting for age, type of laser treatment and baseline visual field MD. The finding of a greater baseline IOP and a greater percentage of IOP reduction at day 1 to be associated with a greater percentage of IOP reduction at 12 months was also observed in the multivariable models when the analysis was limited to PSLT (online supplementary table 3) or SLT (online supplementary table 4).

\section{Safety of SLT/PSLT}

All patients had mild anterior chamber inflammation after PSLT/ SLT which subsided within 1 week to 1 month except for one patient in the PSLT-treated group who developed protracted anterior uveitis which resolved after applying topical steroid for 6 months. One patient treated with SLT and one patient treated with PSLT had IOP increased more than $20 \%$ from baseline at day 1. These patients had IOP remained to be higher than the baseline IOP at 12 months $(11.1 \%$ higher than baseline IOP for the PSLT-treated patient, and 4.5\% higher than baseline IOP for the SLT-treated patient) (figure 4B). At 12 months, three patients, one treated with SLT and two treated with PSLT, had IOP elevation more than $20 \%$ (range: $22.4 \%$ to $27.5 \%$ ) from the baseline. The mean number of topical IOP-lowering medications applied at 12 months was $0.3 \pm 0.6$ and $0.4 \pm 0.6$ for the SLT and PSLT groups
(A)

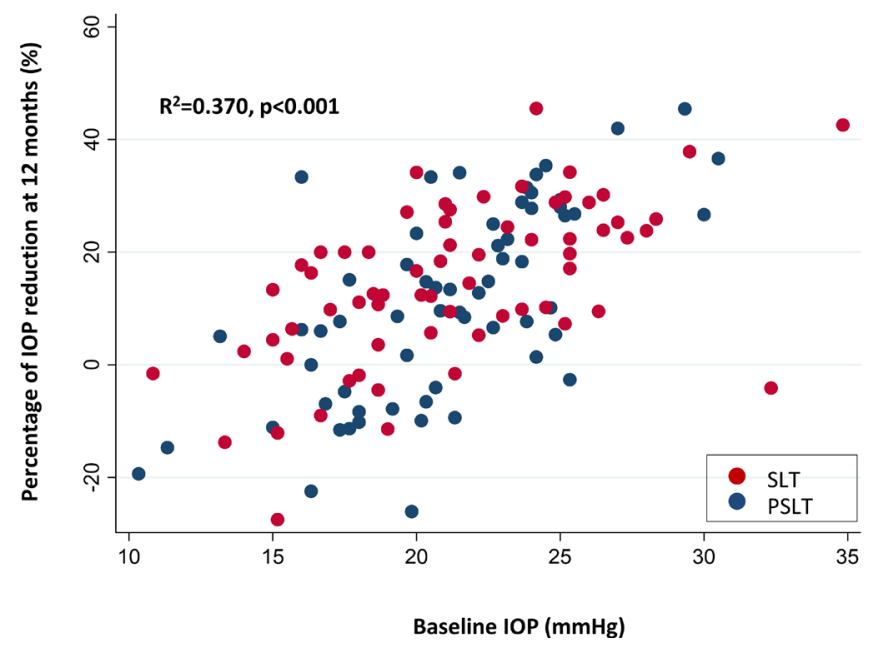

(B)

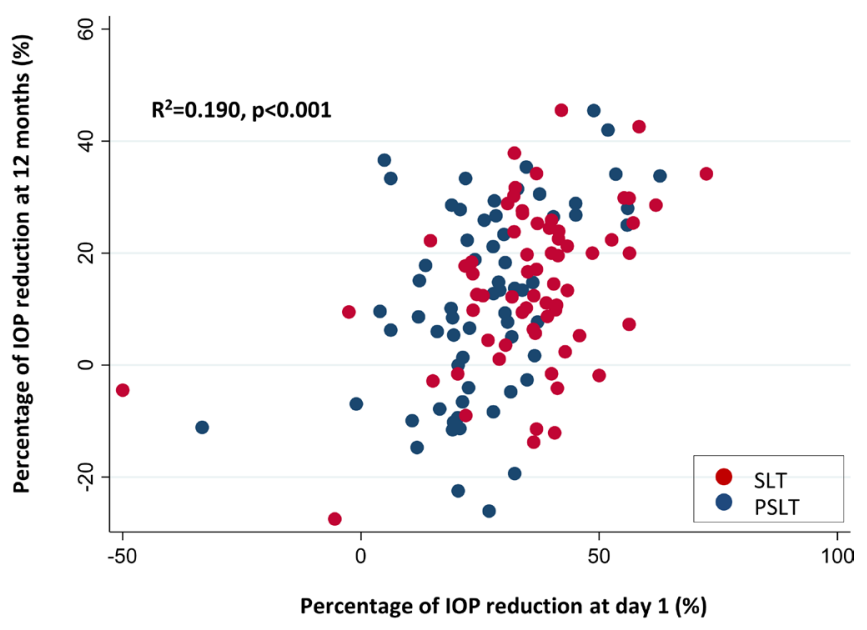

Figure 4 Association of baseline intraocular pressure (IOP) (A) and percentage of IOP reduction at day 1 (B) with the percentage of IOP reduction at 12 months following pattern scanning laser trabeculoplasty (PSLT) or selective laser trabeculoplasty (SLT). 
respectively $(p=0.820)$. In the PSLT-treated group, one patient developed acute conjunctivitis 4 days after the laser procedure which resolved in 2 weeks; one patient received phacoemulsification and intraocular lens implantation at 3.5 months after the laser procedure (the cataract surgery was scheduled before the laser procedure). In the SLT group, one patient developed primary angle closure (without posterior anterior synechiae) at 6 months; laser peripheral iridotomy was performed. Visual field MD, average RNFL thickness, corneal endothelial cell counts and visual acuity were similar between the treatment groups at 12 months (online supplementary table 5 ).

\section{DISCUSSION}

Level I evidence for laser trabeculoplasty is sparse and it has been advocated by the American Academy of Ophthalmology that further research into the differences among the lasers and techniques of treatment used in trabeculoplasty is necessary. ${ }^{11}$ Our clinical trial was designed to address the treatment difference, if any, between PSLT and SLT. IOP measurements throughout the 12-month follow-up were found to be comparable between PSLT and SLT in patients with POAG or OHT (figure 3). The proportion of patients achieving complete success was $25.4 \%$ in the SLT-treated group, and $15.4 \%$ in the PSLT-treated group $(\mathrm{p}=0.155)$. Over $70 \%$ of patients remained medication-free at 12 months. There were no detectable differences in visual field MD, average RNFL thickness, corneal endothelial cell counts and visual acuity between the treatment arms at the baseline and 12-month follow-up. Our data suggest PSLT to be similar in the safety profile, and not superior in the IOP-lowering efficacy compared with SLT in patients with POAG or OHT.

Whereas SLT has been extensively investigated, ${ }^{12-14}$ at the time of writing, there were only three studies reporting the treatment response of PSLT, all have follow-up up to 6 months. ${ }^{5615}$ The percentage of IOP reduction from the baseline ranged from $18.3 \%$ in the study by Espinoza and colleagues (25 patients with open-angle glaucoma $)^{15}$ and $19.1 \%$ in the study by Mansouri and Shaarawy (29 patients with POAG or pseudoexfoliative glaucoma), ${ }^{6}$ to $24 \%$ in the study by Turati and colleagues ( 25 patients with open-angle glaucoma). ${ }^{5}$ Mansouri and Shaarawy reported a greater percentage of IOP reduction in patients treated with PSLT than those treated with SLT at 1 month $(\mathrm{p}<0.01)$ and 3 months $(\mathrm{p}<0.01)$. Complete success, defined as $\geq 20 \%$ IOP reduction without IOP-lowering medication, was $37 \%$ and $26 \%$, respectively, at 6 months $(p=0.09)$. In our study, the percentages of IOP reduction were similar between PSLT and SLT throughout the 12-month follow-up (except for day 1) in 132 patients with POAG or OHT (figure 3). In eyes treated with SLT, the IOP decreased by $14.7 \%$ (95\% CI: $11.2 \%$ to $18.1 \%$ ) at 12 months, which falls within the range of IOP reduction (between 6.9\% and 35.9\%) reported in a systematic review of 35 studies of SLT in patients with open-angle glaucoma who had at least 12 months of follow-up. ${ }^{13}$ Disparities in ethnicity, baseline IOP, follow-up duration and subtypes of glaucoma among the individual studies may contribute to the variation in IOP reduction following SLT. ${ }^{12-14}$ We showed the IOP-lowering effect of PSLT, $11.6 \%$ (95\% CI: $7.4 \%$ to $15.9 \%$ ) reduction at 12 months, to be similar to SLT. The difference in mean IOP between the treatment groups at 12 months was $0.5 \mathrm{~mm} \mathrm{Hg}$ (95\% CI: -0.6 to $1.6 \mathrm{~mm} \mathrm{Hg}$ ); the current sample size has a power of $94 \%$ to detect a difference of $2 \mathrm{~mm} \mathrm{Hg}$ between the treatment groups at 12 months.

Not unexpectedly, a greater baseline IOP was associated with a greater percentage of IOP reduction after PSLT or SLT (figure 4A), which has been shown in previous studies on SLT and ALT. ${ }^{16-21}$ Notably, we also showed that a greater percentage of IOP reduction at day 1 was predictive of the percentage of IOP reduction at 12 months (figure 4B). The two variables, the baseline IOP and the percentage of IOP reduction at day 1 , together explained $50 \%$ (adjusted $\mathrm{R}^{2}=0.50$ ) of the 1 year treatment response for PSLT, and $41 \%$ (adjusted $\mathrm{R}^{2}=0.41$ ) for SLT, after controlling for other covariates (online supplementary tables 3 and 4). Given that the percentage of IOP reduction at day 1 was greater for SLT-treated eyes (35.5\%) than PSLT-treated eyes $(26.1 \%)$, SLT might be more effective in attaining a greater IOP reduction at 12 months. This is reflected from the finding of a greater proportion of patients attaining complete treatment success with SLT (25.4\%) than PSLT (15.4\%) although statistically insignificant. Nevertheless, the finding of baseline IOP and treatment response at day 1 to be predictive of treatment response at 12 months in both PSLT-treated and SLT-treated eyes is relevant to inform treatment prognosis for individual patients.

Although there was a significant IOP spike at day 1 (IOP increase more than $20 \%$ from baseline) in two patients, one in the PSLT group, one in the SLT group and one patient developed anterior uveitis for 6 months, they experienced no clinical consequences. High safety profiles of SLT and PSLT are supported by the lack of significant differences in the visual field MD, average RNFL thickness, corneal endothelial cell counts and visual acuity between the treatment arms at the baseline (online supplementary table 1 ) and the 12 months follow-up (online supplementary table 5).

The goal of glaucoma treatment is to preserve patients' quality of life. The study is limited by not including quality of life measures although 1-year follow-up may not be long enough to capture changes in quality of life. The long-term costeffectiveness of PSLT versus SLT remains unanswered. As the study included only Chinese patients, the study finding may not be generalised to other ethnic groups.

To summarise, our data provide level I evidence supporting PSLT to be a safe and effective means to lower IOP in patients with POAG or OHT although it does not provide additional IOP-lowering compared with SLT. One-year treatment response can be predicted from the baseline IOP and the percentage of IOP reduction at day 1 . Additional studies are needed to address to what degree the IOP-lowering effect of PSLT can be sustained beyond 1 year and whether additional IOP reduction can be attained with repeat PSLT or SLT.

Contributors Study design: CK-SL. Obtained funding: CK-SL. Patient recruitment: TTW, GL, PPC, NCYC, AC, CK-SL. Data collection: TTW, GL, PPC, NCYC, AC, CK-SL, VSMC, GL. Data analysis: TTW, AC, VSMC, GL, CK-SL. Drafting manuscript: TTW, CK-SL.

Funding This study was funded by Hong Kong Food and Health Bureau Health and Medical Research Fund (02130446).

Competing interests CK-SL has received research support in the form of instrument (Cirrus HD-OCT) from Carl Zeiss Meditec, and in the form of research grant, speaker honorarium and instrument (Triton OCT) from Topcon.

Patient consent for publication Not required.

Ethics approval The study was approved by the Kowloon Central Cluster research ethics committee.

Provenance and peer review Not commissioned; externally peer reviewed.

Data availability statement Data are available upon reasonable request. Deidentified participant data will be available upon request from the corresponding author-Christopher Leung (cksleung@cuhk.edu.hk).

\section{ORCID iDs}

Poemen Puiman Chan http://orcid.org/0000-0003-4298-8806

Christopher Kai-Shun Leung http://orcid.org/0000-0003-4862-777X 


\section{REFERENCES}

1 Gazzard G, Konstantakopoulou E, Garway-Heath D, et al. Selective laser trabeculoplasty versus eye drops for first-line treatment of ocular hypertension and glaucoma (LiGHT): a multicentre randomised controlled trial. Lancet 2019;393:1505-16.

2 Anderson RR, Parrish JA. Selective photothermolysis: precise microsurgery by selective absorption of pulsed radiation. Science 1983;220:524-7.

3 Latina MA, Park C. Selective targeting of trabecular meshwork cells: in vitro studies of pulsed and CW laser interactions. Exp Eye Res 1995;60:359-71.

4 Nozaki M, Mansouri K. Pattern scanning laser trabeculoplasty offers benefits over selective laser trabeculoplasty for glaucoma patients. Glaucoma Today 2015.

5 Turati M, Gil-Carrasco F, Morales A, et al. Patterned laser trabeculoplasty. Ophthalmic Surg Lasers Imaging 2010;41:538-45.

6 Mansouri K, Shaarawy T. Comparing pattern scanning laser trabeculoplasty to selective laser trabeculoplasty: a randomized controlled trial. Acta Ophthalmol 2017;95:e361-5.

7 Leung CKS, Lam S, Weinreb RN, et al. Retinal nerve fiber layer imaging with spectraldomain optical coherence tomography: analysis of the retinal nerve fiber layer map for glaucoma detection. Ophthalmology 2010;117:1684-91.

8 Barkana Y, Belkin M. Selective laser trabeculoplasty. Surv Ophthalmol 2007:52:634-54.

9 Shaarawy TM, Sherwood MB, Grehn F, eds. Guidelines on design and reporting of glaucoma surgical trials. Amsterdam, The Netherlands: Kugler Publications, 2009.

10 Hodge WG, Damji KF, Rock W, et al. Baseline IOP predicts selective laser trabeculoplasty success at 1 year post-treatment: results from a randomised clinical trial. Br J Ophthalmol 2005;89:1157-60.
11 Samples JR, Singh K, Lin SC, et al. Laser trabeculoplasty for open-angle glaucoma: a report by the American Academy of ophthalmology. Ophthalmology 2011;118:2296-302.

12 Garg A, Gazzard G. Selective laser trabeculoplasty: past, present, and future. Eye 2018:32:863-76.

13 Wong MOM, Lee JWY, Choy BNK, et al. Systematic review and meta-analysis on the efficacy of selective laser trabeculoplasty in open-angle glaucoma. Surv Ophthalmol 2015;60:36-50.

14 McAlinden C. Selective laser trabeculoplasty (SLT) vs other treatment modalities for glaucoma: systematic review. Eye 2014;28:249-58.

15 Espinoza G, Castellanos L, Rodriguez-Una I, et al. Clinical outcomes of patterned laser trabeculoplasty as adjuvant therapy in open angle glaucoma and ocular hypertension. Int J Ophthalmol 2018;11:635-40.

16 Pillunat KR, Spoerl E, Elfes G, et al. Preoperative intraocular pressure as a predictor of selective laser trabeculoplasty efficacy. Acta Ophthalmol 2016;94:692-6.

17 Ayala M, Chen E. Predictive factors of success in selective laser trabeculoplasty (SLT) treatment. Clin Ophthalmol 2011:5:573-6.

18 Johnson PB, Katz LJ, Rhee DJ. Selective laser trabeculoplasty: predictive value of early intraocular pressure measurements for success at 3 months. Br J Ophthalmol 2006;90:741-3.

19 Singh D, Coote MA, O'Hare F, et al. Topical prostaglandin analogues do not affect selective laser trabeculoplasty outcomes. Eye 2009;23:2194-9.

20 Heijl A, Peters D, Leske MC, et al. Effects of argon laser trabeculoplasty in the early manifest glaucoma trial. Am J Ophthalmol 2011;152:842-8.

21 Garg A, Vickerstaff V, Nathwani N, et al. Primary selective laser trabeculoplasty for open-angle glaucoma and ocular hypertension: clinical outcomes, predictors of success, and safety from the laser in glaucoma and ocular hypertension trial. Ophthalmology. In Press 2019;126:1238-48. 